\title{
Interactive Information Retrieval: Context and Basic Notions
}

\author{
David Robins \\ Louisiana State University, \\ School of Library and Information Science
}

drobin2@lsu.edu

\begin{abstract}
This paper provides an introduction to interactive information retrieval--the study of human interaction with information retrieval systems. Interactive information retrieval may be contrasted with the "system-centered" view of information retrieval in which changes to information retrieval system variables are manipulated in isolation from users in laboratory situations. The paper elucidates current models of interactive information retrieval, namely, the episodic model, the stratified model, the interactive feedback and search process model, and the global model of polyrepresentation. Future directions for research in the field are discussed.
\end{abstract}

Keywords: interactive information retrieval; information retrieval; interactive models

\section{Introduction}

Information retrieval (IR) is a discipline concerned with the processes by which queries presented to information systems are matched against a "store" of texts (the term text may be substituted with still images, sounds, video clips, paintings, or any other artifact of intellectual activity). The end result of the matching process is a listing of texts that are a subset of the total store. Any number of means may accomplish the matching process, but essentially, when specified attributes in a query are found to correspond with specified attributes of a text, the text is included in the listing.

Since the middle of the 20th century, most efforts to improve information retrieval have focused on methods of matching text representations with query representations. Recently, however, researchers have undertaken the task of understanding the human, or user, role in IR. The basic assumption behind these efforts is that we cannot design effective IR systems without some knowledge of how users interact with them. Therefore, this line of research that studies users in the

Material published as part of this journal, either on-line or in print, is copyrighted by the publisher of Informing Science. Permission to make digital or paper copy of part or all of these works for personal or classroom use is granted without fee provided that the copies are not made or distributed for profit or commercial advantage AND that copies 1) bear this notice in full and 2) give the full citation on the first page. It is permissible to abstract these works so long as credit is given. To copy in all other cases or to republish or to post on a server or to redistribute to lists requires specific permission and payment of a fee. Contact Editor@inform.nu to request redistribution permission. process of directly consulting an IR system is called interactive information retrieval (IIR).

In order to understand the context in which IIR has developed, I will give a brief background on traditional IR studies. I will follow this with a description of current models of IIR, and then conclude with a discussion of new directions in IIR.

\section{Background: The System Approach}

The system approach to IR has grown out of concerns with the "library problem" (e.g., Maron \& Kuhns, 1960, p. 217), the problem of searching and retrieving relevant documents from IR systems. The hardware and software problems associated with document retrieval and document representation still persist. The development of digitally based IR systems requires computer programs that match requests with stores of documents, and then produce output. In sophisticated systems of this sort, both input terms, and output text, may be ranked according to preset criteria. The challenge to researchers in this area is to develop algorithms that optimize such rankings.

There are, however, difficulties with the system orientation to IR. The first problem with the system view is in how IR systems are evaluated. In the system approach to information retrieval, system effectiveness is calculated by two measures: recall and precision. For any given search on a given database, recall is the ratio of the number of relevant documents retrieved to relevant documents in the database. Precision is the ratio of the number of relevant documents retrieved to the number of documents retrieved. These measurements rest on 


\section{Interactive Information Retrieval}

the assumptions that: (a) all documents in the system are known; (b) all documents in the system can be judged in advance for their usefulness (relevance) for any given problem; and (c) users' relevance judgments are a single event based solely on a text's content. Assumption (a) is valid only in the case of small test collections. Assumptions (b) and (c) are based on static notions relevance. A user's judgment of the usefulness of a document may vary with respect to his or her information seeking stage (Kuhlthau, 1991), criteria other than the topic of the document such as availability of the text (Barry, 1994), or his or her ability to express the information need to an intermediary or to an IR system (Belkin, 1980; Taylor, 1968).

The second difficulty with the system approach is that language is treated as if it were precise. Although natural language processing systems have made tremendous strides in the past decade (Turtle, 1994), language will remain a problem for system designers. The reason for this is that language can be best understood by how it is used, rather than by what is said (Blair, 1990). In other words, it may be possible to understand more about what a user says to an intermediary if his or her motives or goals are understood. Words taken on their own account may be misleading. For example, in a library reference situation, a user may request directions to the current periodicals. The librarian, being experienced with such requests, may encourage the user to consult an index if s/he has a specific problem. However, the user may only be there to meet a friend, rather than to seek information. Parsing algorithms can only go so far in sorting out what a person means, versus what they say.

Finally, human interaction with information systems is largely ignored. In some systems oriented research (e.g., Salton, 1972), queries are constructed for use in the experiment (on a small, experimental database), and search output is judged for relevance by a paid assistant, or the like. In reality, a user has some stake in the outcome of the search. Because of that stake, a real user is able to reformulate queries based on his or her domain knowledge, and interest in completing a task. Depending on what is at stake, a real user may iteratively query the system until s/he is sure that s/he has made a comprehensive search. Depending on what stage of a particular project $\mathrm{s} / \mathrm{he}$ is working, different types of queries may be formed for the same general topic. A more complex set of circumstances is realized when information problems are considered to be part of the query and relevance judgment process, than when queries are sliced away from them.

In response to the inadequacies of the system approach, researchers began looking at the other side of the equation in IR: human beings who use IR systems. During the 1970s, a research program began to establish itself around user-related concerns. Examples of such concerns are information needs and uses (Dervin \& Nilan, 1986), motivations for information behavior (Belkin, 1980), and IR interaction (Saracevic \& Kantor, 1988; Fidel, 1985; Belkin, Brooks \& Daniels, 1987).

IR interaction is a promising research paradigm that stresses the iterative nature of information searching. That is, it seeks to better understand phenomena such as search strategies, search term generation and use, and successive searches by users for the same research problem. Methods employed to study these phenomena include observation of users in naturalistic settings, discourse analysis, and other protocol analyses such as think aloud protocols. The focus of much of today's research is to gain an understanding of end-user and mediated searching that will guide the development of "intelligent" IR systems that will act as guides to information searching to end users. In the next section I will describe some models that have been developed out this line of research.

\section{Interactive IR: Current Models}

Traditional models of information retrieval interaction describe only minimally the dynamic nature of this phenomenon. In this section, four models that attempt to describe further the ways in which IR interaction is dynamic are explored. These four models are: (i) Saracevic's (1997) stratified model of interactive IR; (ii) Belkin's (1996) episodic model of IR interaction; (iii) Spink's (1997) interactive feedback and search process model; and (iv) Ingwersen's (1996) global model of polyrepresentation.

Each of these models presents an alternative view to the traditional model of information retrieval. The traditional model of IR is based on the notion that IR takes place on two separate tracks, that is, system and user. Essentially, the traditional model of IR holds that IR systems are comprised of texts that are represented and organized to facilitate retrieval. On the other side of the model, users approach IR systems with some information problem/need that they represent in the form of a research question that must be further reduced to a query. These two tracks meet at the point where queries and organized files are compared. The results of the comparison (i.e., system output) are presented to the user (i.e., feedback) in the form of IR system output.

The inadequacies of this model have been exposed by IR interaction research. First, the traditional model does not account for the complexities of interaction: (i) among humans (e.g., users and search intermediaries); (ii) between humans and IR systems and the iterative nature of such; (iii) with respect to feedback as shown by Spink (1993; 1997). Similarly, the model does not account for complexities exhibited by indexers and organization schemes as shown by past research (Leonard, 1975). In order to address these inadequacies, a small but growing number of researchers are beginning to 
directly address the problems of interaction in IR. The three mentioned above are elucidated in the following sections.

The stratified model of IR interaction (Saracevic, 1997) includes the two-track aspect of the traditional model, shown by the two arrows indicating adaptation. The difference is that this model accounts for multiple dimensions of user involvement in IR processes. That is, Saracevic's model accounts for user environment and situation, in addition to user knowledge, goals, intent, beliefs, and tasks. This model improves on the traditional model by showing the complexity of a user's environment. One potential weakness of the model lies in its lack of description of temporal effect. Saracevic notes "during IR interaction, as it progresses, these deeper level cognitive and situational aspects in interaction can and often do change problem or question is redefined, refocused, and the like" ( $\mathrm{p}$. 7). Notwithstanding, no mention of the effects time and iteration is included in his model. The following two models do bring the dimension of time into their treatment of interactive IR.

Belkin's notions of IR interaction are based on his commitment to his anomalous states of knowledge hypothesis (ASK) (Belkin, 1980), which essentially states that users come to the search tasks with varying degrees of knowledge regarding the subject of their search. ASK was also the basis for

MONSTRAT (Belkin, Brooks, \& Daniels, 1987), which attempted to create the basis for an IR system from the study of user modeling behavior by search intermediaries. The episodic model, however, represents a significant advance over MONSTRAT. Two major improvements over MONSTRAT are present in the episodic model. First, the model shows how many of the same events in IR interaction repeat themselves. In this way, the repeating frames display the cyclic, temporal nature of IR interaction. Second, the episodic model reorganizes the nine MONSTRAT functions into two groups. What Belkin's model lacks, however, is a treatment of the social/environmental facets of user information problems. User's tasks and goals are mentioned, but there is no mention of the setting from which these tasks and goals are causally derived. Nevertheless, the episodic model represents a stride forward in providing a research framework for interactive IR.

Of the current models of IR interaction, Spink's (1997) interactive feedback and search process model offers the most comprehensive coverage of the complex, cyclical nature of IR interaction. Spink $(1993 ; 1997)$ has studied the nature of feedback in information retrieval, and thus, is concerned with iteration and periodicity in IR interaction. Her research on the concept of feedback has brought cybernetics and systems theory into the realm of information science. Spink's (1997) feedback model accounts for time as a factor in IR interaction, as well as the cycles that occur during searches. The ongoing element at the top of the model is search process and strategies, hence, time. These processes involve any number of cy- cles. Cycles are defined as processes completed between each search command; that is, the time and processes between a query (terms typed/combined and entered) and the next query reformulation (again, terms typed/combined and entered). During each cycle, any number of interactive feedback loops may occur. These feedback loops consist of discussions between user and search intermediary regarding either: (i)content relevance; (ii) term relevance; (iii) magnitude relevance; (iv) tactical review; or (v) term review. Therefore, an interactive feedback loop would be delineated when one of the participants gives feedback to the other regarding one of the five topics above, after which some judgment or action is taken. Spink's (1997) feedback model has the strength of suggesting the cyclical nature of IR interaction. A weakness of the model is its lack of accounting for cognitive changes, or processes. We see that tactics, moves, and judgments are included, but there is no means of connecting those processes to changes in the search, such as alternative tactics as a result of a feedback loop.

Finally, Ingwersen (1996) work synthesizes much of the models reviewed in this section so far. He attempts to model IR processes from a global perspective. A global perspective holds that all of the factors that influence and interact with a user, such as search intermediary, IR system, and texts, should be considered in IR research. The design variables put forth by Ingwersen show the wide ranging influence of factors such as social environment, IR system, information objects, intermediary, and user. Ingwersen incorporates these design variables into the notion of polyrepresentation, which may be summarized as follows:

- redundancy is inherent in information retrieval processes

- examples of this form of redundancy are:

- identical documents retrieved from different search engines or databases

- identical documents retrieved from different searches at different points in time

- cognitive overlap is the term for identical items retrieved in the above scenarios

- this redundancy presents an opportunity to increase retrieval effectiveness

In other words, documents retrieved by multiple searches have a higher probability of usefulness to a user.

User cognitive space is divided into four components: Information Need; Problem Space; Current Cognitive State; and Work-Task/Interest Domain. Information need is characterized by the ability of a user to state specifically what s/he would like to retrieve from an information system during a particular search. Problem space is defined in terms of a user's uncertainty with regard to his/her search. Problem space may be thought of as the gap between what a user knows (current cognitive state) and his/her ability to express an information need (Belkin, 1980). Current Cognitive State is defined as 


\section{Interactive Information Retrieval}

what a user knows (or at least thinks s/he knows) at a given point in time, and is characterized by certainty of such knowledge. Finally, the Work-Task/Interest Domain is the set of environmental and social constraints under which a user seeks information. These constraints tend to be static in nature, according to Ingwersen (1996). Examples are projects such as dissertations or term papers that require background research, and social environments such as graduate school or a business setting.

Ingwersen $(1996 ; 1992)$ has presented a reasonably complete synthesis of research and thought regarding IR interaction. The main problem with Ingwersen's (1996) approach is how to get input from user cognitive space into the request model builder. The differences among the four components of user cognitive space are subtle. For example, if a user expresses information need from the standpoint of current knowledge, it is unclear whether the user is stating information need, or current cognitive state. Therefore, constructing the proper request model builder may be difficult, if even possible. Nevertheless, Ingwersen has presented a model that has "plausible validity." His models are based on solid ground, both conceptually, and with empirical evidence. However, the empirical evidence on which his hypotheses are based represent syntheses of many different studies, only one of which was done by Ingwersen. This is not necessarily a negative, but it is a caveat when studying Ingwersen.

The four models presented here represent some of the most well formed conceptions of interactive IR processes. They do not represent a complete list of such models, but only provide an introduction. In addition, they do not constitute the "final word" on interactive IR. More research is needed in this growing body of knowledge.

\section{Conclusions and Future Directions}

Interactive IR provides a rich research agenda. It includes human behavior elements, but it also requires a basic understanding of how IR systems work so that realistic IIR researchers can collaborate with IR system designers. The future of IIR is in interdisciplinary collaboration and research. the problem is too large for any one field to cover. Researchers from disciplines, such as psychology, sociology, business, computer science, library and information science, and communications, to name a few, are needed to give full description to IIR. By doing so, IIR research will expand into areas such as domain analysis to determine the effects of organizations and disciplines on IR interaction. Other areas of research that have only been recently opened include successive search models (Spink, Wilson, Ellis \& Ford, 1998), studies of the impact of partial relevance judgments by users (Spink, Griesdorf \& Bateman, 1997), question asking (i.e. elicitation) behavior by participants in searches (Wu, 1993; Spink, Goodrum \& Robins, 1998), shifts of topic focus (Robins, 1998) and search strategy (Xie, 1997), and end user searching on the Web (Jansen, Spink, Bateman \& Saracevic, 1998)

IIR is a relatively new, but fruitful, area of interest to the IR community. As a more diverse set of researchers enter the playing field, more studies should support the drive for more intelligent IR systems. Two of the project descriptions within the National Science Foundation's Information Technology Research Program (NSF 99-167) deal with human computer interfaces, and the social and economic impact of information systems. Both of these areas indicate the importance placed by the NSF on the larger picture of information technology: that of the human side which is addressed by IIR research.

\section{References}

Barry, C. L. (1994). User-defined relevance criteria: An exploratory study. Journal of the American Society for Information Science, 45(3), 149-159.

Belkin, N. J. (1980). Anomalous states of knowledge as a basis for information retrieval. Canadian Journal of Information Science, 5 , 133-143.

Belkin, N. J. (1996) Intelligent information retrieval: Whose intelligence? In: ISI '96: Proceedings of the Fifth International Symposium for Information Science. Konstanz: Universtaetsverlag Konstanz, 25-31.

Belkin, N. J., Brooks, H. M. \& Daniels, P. J. (1987). Knowledge elicitation using discourse analysis. International Journal of ManMachine Studies, 27, 127-144.

Blair, D. C. (1990). Language and representation in information retrieval. Amsterdam: Elsevier.

Dervin, B. \& Nilan, M. (1986). Information needs and uses. Annual Review of Information Science and Technology, 21, 3-33.

Fidel, R. (1985). Moves in online searching._Online Review, 911), 61-74.

Ingwersen, P. (1996). Cognitive perspectives of information retrieval. Journal of Documentation, 52(1), 3-50.

Jansen, B.J., Spink, A., Bateman, J. \& Saracevic, T. (1998). Searchers, the subjects they search, and sufficiency: A study of a large sample of EXCITE searches. In Proceedings of Webnet 98.

Kuhlthau, C. C. (1991). Inside the search process: Information seeking from the user's perspective. Journal of the American Society for Information Science, 42(5), 361-371.

Leonard, L. E. (1975). Inter-indexer consistency and retrieval effectiveness: Measurement of relationships. Unpublished doctoral dissertation, University of Illinois, Champaign-Urbana.

Maron, M. E. \& Kuhns, J. L. (1969). On relevance, probabilistic indexing and information retrieval. Journal of the Association for Computing Machinery, 7, 216-244. 
NSF 99-167 (1999). Information Technology Research Program. http://www.nsf.gov/pubs/1999/nsf99167/nsf99167.htm.

Robins, D. (1998a). Dynamics and dimensions of user information problems as foci of interaction in information retrieval. ASIS '98: Proceedings of the 61st ASIS Annual Conference, 35, 327-341.

Salton, G. (1972). A new comparison between conventional indexing (MEDLARS) and automatic text processing (SMART). Journal of the American Society for Information Science, 23, 75-84.

Saracevic, T. (1997). The stratified model of information retrieval interaction: Extension and application. Proceedings of the 60th Annual Meeting of the American Society for Information Science, 34, 313327.

Saracevic, T. \& Kantor, P. (1988). A study of information seeking and retrieving. III. Searchers, searches, and overlap. Journal of the American Society for Information Science, 39(3), 197-216.

Spink, A. (1993). Feedback in information retrieval. Unpublished doctoral dissertation, Rutgers University.

Spink, A. (1997). Study of interactive feedback during mediated information retrieval. Journal of the American Society for Information Science, 48(5), 382-394.

Spink, A., Goodrum, A. \& Robins, D. (1998). Elicitation behavior during mediated information retrieval. Information Processing and Management, 34(2/3), 257-273.
Spink, A. \& Greisdorf, H. (1997). Partial Relevance Judgments During Interactive Information Retrieval: An Exploratory Study. Sixtieth Annual Meeting of the American Society for Information Science, 34, 111-122.

Spink, A., Wilson, T. D., Ellis, D. \& Ford, N. (1998). Modeling users' successive searches in digital environments. D-Lib Magazine, (April, 1998), http://www.dlib.org/dlib/april98/04spink.html.

Taylor, R. S. (1968). Question-negotiation and information seeking. College and Research Libraries, 29, 178-194.

Turtle, H. (1994). Natural language vs. Boolean query evaluation: A comparison of retrieval performance. In W. B. Croft \& C. J. van Rijsbergen (Eds.), SIGIR '94: Proceedings of the seventeenth annual international ACM-SIGIR conference on research and development in information retrieval (pp. 212-220). London: SpringerVerlag.

Wu, M. M. (1993). Information interaction dialog: A study of patron elicitation in the information retrieval interaction. Unpublished doctoral dissertation, Rutgers University.

Xie, Hong (1997). Planned and situated aspects in interactive IR: Patterns of user interactive intentions and information seeking strategies. Proceedings of the $60^{\text {th }}$ Annual Meeting of the American Society for Information Science, 34, 101-110. 